\title{
Montagens curtas com instrumentação do nível fracturado e moldagem in situ no tratamento das fracturas tipo "burst" da coluna toracolombar
}

\author{
Short-segment fixation of thoracolumbar burst fractures using pedicle \\ fixation at the level of the fracture and in situ bending \\ Montajes cortas con instrumentación del nivel fracturado y molde \\ in situ en el tratamiento de las fracturas tipo burst de la columna \\ toracolumbar
}

\author{
Pedro Cacho Rodrigues ${ }^{1}$ \\ Manuel Ribeiro da Silva' \\ Vítor Vidinha' \\ Nuno Neves' \\ Rui Matos ${ }^{1}$ \\ Rui Pinto'
}

\section{RESUMO}

Objectivo: Avaliação dos resultados clínicos e radiográficos do tratamento cirúrgico de fracturas tipo burst da coluna toracolombar por fixação posterior curta, com instrumentação do nível fracturado e moldagem in situ. Métodos: Entre Novembro de 2007 e Janeiro de 2009, foram seleccionados pacientes que sofreram fractura da coluna toracolombar tipo burst a um nível, com presença de instabilidade neurológica ou mecânica, submetidos a fixação posterior curta com instrumentação do nível fracturado e moldagem in situ. Foi realizada avaliação radiográfica e clínica no pré-operatório, no pós-operatório imediato e, pelo menos, um ano após a cirurgia. Resultados: Foram incluídos 12 pacientes com uma média de idades de 39,1 anos. O seguimento médio foi de 14,5 meses. Um paciente abandonou a consulta aos dois meses, e dois não compareceram à consulta de avalia-

\section{ABSTRACT}

Objective: Evaluation of clinical and radiographic results of the surgical treatment of thoracolumbar burst fractures with short posterior fixation, including the fractured vertebra and in situ bending. Methods: From November 2007 to January 2009, the authors reviewed patients with traumatic thoracolumbar burst fractures of a single vertebral level, with neurological or mechanical instability, and surgically treated with short posterior fixation including the fractured level and in situ bending. Clinical and radiographic evaluations were performed before, immediately after surgery, and at least at one year after surgery. Results: Twelve patients were included with an average age of 39.1 years. The average follow-up was 14.5 months. One patient was lost to followup at two months, and two were lost to clinical evaluation. Two patients presented
\end{abstract}

\section{RESUMEN}

Objetivo: evaluación de los resultados clínicos y radiológicos del tratamiento quirúrgico de fracturas tipo burst de la columna toracolumbar por fijación posterior corta, con instrumentación del nivel fracturado y molde in situ. Métodos: entre Noviembre de 2007 y Enero de 2009, fueron seleccionados pacientes que sufrieron fractura de la columna toracolumbar tipo burst a un nivel, con presencia de inestabilidad neurológica o mecánica, sometidos a la fijación posterior corta con instrumentación del nivel fracturado y molde in situ. Se realizó evaluación radiográfica y clínica en el pre operatorio, en el postoperatorio inmediato y por lo menos un año después de la cirugía. Resultados: fueron incluidos 12 pacientes con un promedio de edad de 39.1 años. El seguimiento promedio fue de 14.5 meses. Un paciente abandonó la consulta a los dos meses, $y$ dos no comparecieron a la consulta

\footnotetext{
Trabalho realizado no Hospital de São João da Faculdade de Medicina da Universidade do Porto - Portugal.

'Membros do Grupo da Coluna do Serviço de Ortopedia do Hospital de São João da Faculdade de Medicina da Universidade do Porto - FMUP - Portugal
} 
ção clínica. Dois pacientes apresentavam défices neurológicos à entrada (Frankel B). Obtivemos uma melhoria da angulação vertebral de $14,2^{\circ}$, da deformidade cifótica de $11,2^{\circ}$ e $27,2 \%$ de recuperação da altura vertebral anterior. Ao tempo de seguimento final, verificaram-se perdas de $2,7^{\circ}, 3,8^{\circ}$ e $6,1 \%$, respectivamente. Registou-se um Oswestry Disability Index (ODI) médio de 6,2 e uma Visual Analogic Scale (VAS) de 1,6. Os dois pacientes com lesões neurológicas melhoraram para um nível D de Frankel. Não se observou qualquer caso de desmontagem ou falência do material. Conclusões: A instrumentação do nível da fractura aumenta a rigidez da montagem, protegendo a vértebra fracturada de cargas anteriores, garantindo um ponto de fixação adicional que permite uma melhor correcção por moldagem in situ. Os resultados obtidos, radiográficos e clínicos, são bons e mantêm-se ao longo do tempo.

DESCRITORES: Fraturas da coluna vertebral/cirurgia; Fraturas da coluna vertebral/ instrumentação; Vértebras torácicas/lesões; Vértebras lombares/lesões; Cifose/ cirurgia; Fixação de fratura/ métodos with neurological deficits (Frankel B).We achieved an improvement of $14.2^{\circ}$ of vertebral angulation, $11.2^{\circ}$ of kyphotic deformity and a restoration of $27.2 \%$ of anterior vertebral height. At final follow up $2.7^{\circ}, 3.8^{\circ}$ and $6.1 \%$ were lost, respectively. We obtained an average Oswestry Disability Index (ODI) of 6.2 and a Visual Analogic Scale (VAS) of 1.6. The two patients with neurological deficits improved to a level D of Frankel. There was no instrumentation failure. Conclusion: Segmental fixation with instrumentation of the level of the fracture increases construct stiffness and gives additional vertebral body protection from anterior loads, achieving an additional point of fixation that allows a better kyphosis correction by in situ bending. Our clinical and radiographic results are good and long-lasting.

KEYWORDS: Spinal fractures/ surgery; Spinal fractures/ instrumentation; Thoracic vertebrae/injuries; Lumbar vertebrae/injuries; kyphosis/ surgery; Fracture fixation/ methods de evaluación clínica. Dos pacientes presentaron déficits neurológicos a la entrada (Frankel B). Obtuvimos una mejoría del ángulo vertebral de $14.2^{\circ}$, de la deformidad cifótica de $11.2^{\circ}$ y el $27.2 \%$ de recuperación de la altura vertebral anterior. Al tiempo de seguimiento final, se verificaron pérdidas de $2.7^{\circ}, 3.8^{\circ}$ y el $6.1 \%$, respectivamente. Se registró un Oswestry Disability Index (ODI) promedio de 6.2 y una Escala Analógica Visual (VAS) de 1.6. Los dos pacientes con lesiones neurológicas mejoraron para un nivel $D$ de Frankel. No se observó cualquier caso de desmontaje o falencia del material. Conclusiones: la instrumentación del nivel de la fractura aumenta la rigidez del molde, protegiendo la vértebra fracturada de cargas anteriores, garantizando un punto de fijación adicional que permite una mejor corrección por molde in situ. Los resultados obtenidos, tanto los radiográficos cuanto los clínicos, son buenos y se mantienen a lo largo del tiempo.

DESCRIPTORES: Fracturas de la columna vertebral/cirugía; Fracturas de la columna vertebral/instrumentación; Vértebras torácicas/lesiones; Vértebras lumbares/lesiones; Cifosis/cirugía; Fijación de fractura/métodos

\section{INTRODUÇÃO}

As fracturas tipo burst da coluna toracolombar representam de 10 a $20 \%$ de todas as fracturas vertebrais ${ }^{1-3}$.

Um dos objectivos do tratamento é minimizar o número de níveis instrumentados sem comprometer a estabilidade da montagem, de modo a preservar a mobilidade ${ }^{4-8}$.

Desde que Holdsworth descreveu, em 1963, pela primeira vez esse tipo de fracturas ${ }^{9}$, desenvolveram-se várias opções de tratamento cirúrgico, incluindo parafusos pediculares e barras por via posterior, o recurso à via anterior ou ambos. No que respeita à fixação por via posterior, existem várias soluções que variam no número de níveis instrumentados (montagens curtas ou longas) e na inclusão ou não do nível fracturado na montagem (segmentar ou não-segmentar, respectivamente). As instrumentações posteriores não-segmentares resultam em taxas de falência elevadas, variando entre 5 e $94 \%{ }^{10-16}$. Causas prováveis incluem a osteoporose, a falta de suporte da coluna anterior e insuficientes pontos de fixação ${ }^{17}$.

Contrariamente ao que acontece nas fracturas de flexão-distracção ou fracturas-luxação, na maioria das fracturas tipo burst, a coluna posterior permanece intac$\operatorname{ta}^{18}$. Logo, a montagem segmentar, com instrumentação do nível fracturado, fornece conceptualmente um ponto adicional de fixação, suporte indirecto da coluna anterior traduzindo-se numa vantagem biomecânica ${ }^{17}$.

O objectivo deste estudo foi avaliar o papel e os resultados das montagens curtas com instrumentação do nível fracturado e moldagem in situ no tratamento das fracturas tipo burst da coluna toracolombar.

\section{MÉTODOS}

Foi realizado estudo retrospectivo em pacientes que sofreram fractura da coluna toracolombar tipo burst a um nível, de Novembro de 2007 a Janeiro 2009. 
Os critérios de inclusão para o estudo consistiram em pacientes com maturação esquelética que: sofreram uma fractura entre T2 e L5, classificada como tipo A3 segundo classificação AO/ASIF e que tivessem sido tratadas cirurgicamente com instrumentação posterior curta segmentar com moldagem in situ.

As indicações cirúrgicas foram a presença de instabilidade neurológica e/ou instabilidade mecânica, traduzida por angulação vertebral $>20^{\circ}$, compromisso do canal vertebral $>50 \%$ e/ou perda de altura anterior do corpo vertebral $>50 \%$.

\section{TÉCNICA CIRÚRGICA}

O paciente é posicionado em decúbito ventral e a mesa é quebrada para ajudar na redução (Figura 1). Os elementos posteriores são expostos por via mediana clássica. Procede-se à instrumentação transpedicular bilateral dos níveis acima e abaixo da fractura e, pelo menos, unilateral do nível fracturado com parafusos pediculares monoaxiais. Promove-se distracção para redução indirecta da coluna média por ligamentotaxia, bloqueiam-se os parafusos e faz-se moldagem in situ (Figura 2), o que permite ganhar altura anterior e uma melhor correcção da cifose.

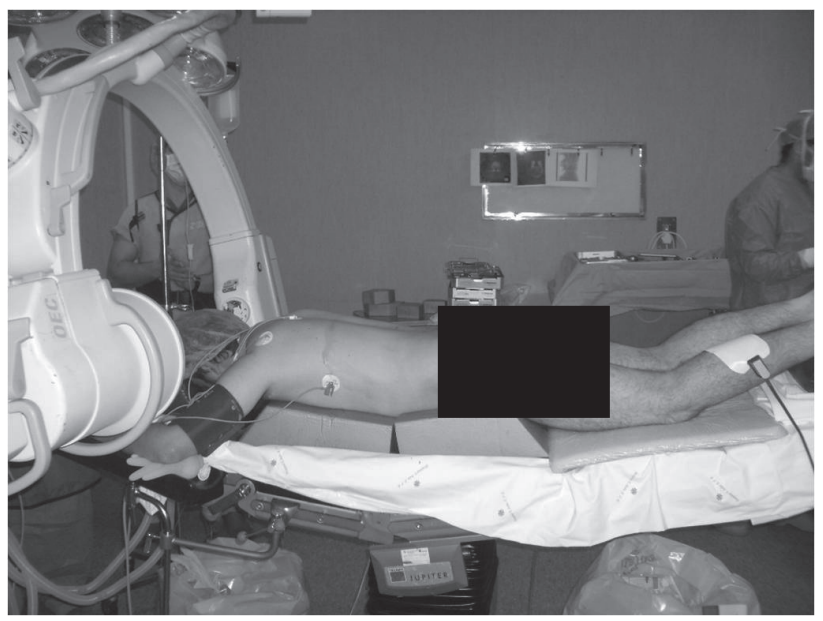

Figura 1

Posicionamento do paciente com quebra da mesa para auxiliar na correcção.

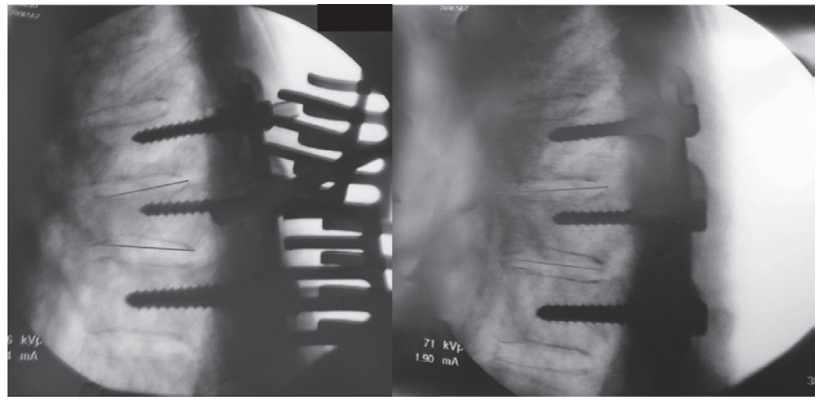

Figura 2

A moldagem in situ permite ganhar altura anterior e melhor correcção da cifose.

\section{MEDIÇÕES RADIOGRÁFICAS}

Realizou-se uma avaliação radiográfica no pré, no pósoperatório imediato e pelo menos um ano após a cirurgia (Figura 3). As radiografias de seguimento foram realizadas em ortostatismo.

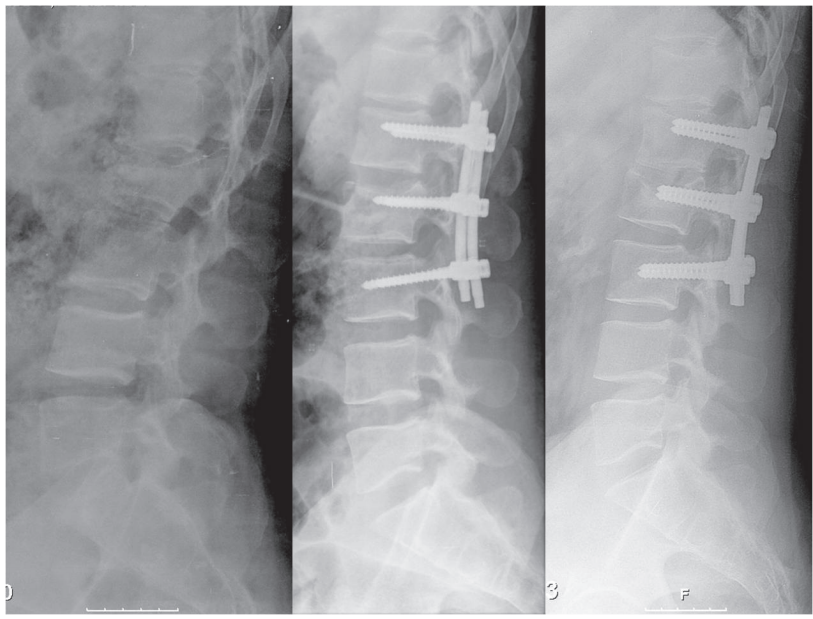

Figura 3

EMFS, 34 anos. Fractura de L2, com angulação cifótica de $10,7^{\circ}$ e mais de $50 \%$ de perda de altura anterior do corpo vertebral. Correcção para 2,2 $2^{\circ}$ de lordose e $95 \%$ de altura vertebral. Ao final de um ano, a perda verificada é mínima.

Foram registadas a angulação de cifose vertebral (entre o prato superior e inferior da vértebra fracturada); a deformidade cifótica (medida entre o prato inferior de vértebra adjacente superior e o prato inferior da vértebra adjacente inferior) e a percentagem de altura anterior do corpo vertebral (razão entre altura anterior e posterior do nível fracturado).

O compromisso do canal vertebral foi avaliado por tomografia computadorizada.

\section{AVALIAÇÃO CLÍNICA}

A instabilidade neurológica foi qualificada segundo a escala de Frankel ${ }^{19}$.

$\mathrm{Na}$ data da avaliação clínica final foram aplicados a escala visual analógica da dor Visual Analogic Scale (VAS) e o índice Oswestry Disability Index (ODI).

\section{RESULTADOS}

Foram selecionados 12 pacientes, 9 do sexo masculino e 3 do feminino (Tabela 1). Observou-se média de idade de 39,1 anos (entre 14 e 60). Os mecanismos de lesão foram queda em altura, em 11 pacientes; e acidente de viação em um paciente. Os níveis atingidos foram T9 (1), T12 (4), L1 (3), L2 (2) e L3 (2). Registaram-se défices neurológicos à entrada em dois pacientes (Frankel B), 17\% do total. Diagnosticaram-se fracturas associadas em 30\% dos pacientes.

A cirurgia ocorreu em média 36h (um dia e meio) após o acidente, e o seguimento médio foi de 14,5 meses (entre 12 e 18). 
TABELA 1 - Resultados da avaliação

\begin{tabular}{lcccccccccccccccc}
\hline & \multicolumn{11}{c}{ Angulação vertebral } & \multicolumn{1}{c}{ Angulação cifótica } & \multicolumn{1}{c}{ Altura do corpo (\%) } \\
\hline Pacientes & Nível & Frankel & Pré & Pós & Final & Perda & Pré & Pós & Final & Perda & Pré & Pós & Final & Perda & ODI & VAS \\
\hline 1 & L2 & E & 21,1 & 1,4 & 7,2 & 5,8 & 10,7 & $-8,7$ & $-2,2$ & 6,5 & 46 & 95 & 86 & -9 & 4 & 0 \\
2 & T9 & B & 15 & 7,5 & 10,5 & 3 & 11 & 13 & 13,5 & 0,5 & 65 & 73 & 70 & -3 & 0 & 2 \\
3 & T12 & E & 27 & 11,2 & 12,5 & 1,3 & 11 & $-1,6$ & 1,4 & 3 & 59 & 82 & 72 & -10 & 12 & 3 \\
4 & L1 & E & 21,7 & 8,4 & 13,5 & 5,1 & 7 & -8 & 2,9 & 10,9 & 54 & 83 & 79 & -4 & 18 & 5 \\
5 & L2 & E & 23,5 & 1,9 & 2,7 & 0,8 & 2,1 & $-6,6$ & $-2,2$ & 4,4 & 64 & 88 & 82 & -6 & 10 & 0 \\
6 & T12 & E & 25,9 & 5,9 & 6,1 & 0,2 & 12,3 & -1 & 2,1 & 3,1 & 52 & 87 & 81 & -6 & 6 & 0 \\
7 & L3 & E & 21 & 1,3 & 2,4 & 1,1 & 3,4 & -18 & $-17,8$ & 0,2 & 57 & 96 & 95 & -1 & & \\
8 & L1 & E & 20,3 & 9 & 11,5 & 2,5 & 7,7 & $-1,2$ & 5 & 6,2 & 68 & 80 & 75 & -5 & 6 & 1 \\
9 & L1 & E & 21 & 4,5 & 11 & 6,5 & 10 & 1,2 & 3,6 & 2,4 & 56 & 94 & 79 & -15 & 0 & 1 \\
10 & L3 & E & 21 & 5,9 & 6,9 & 1 & 0 & $-19,9$ & $-15,5$ & 4,4 & 48 & 88 & 86 & -2 & & \\
11 & T12 & B & 10,6 & 7,8 & 6,6 & $-1,2$ & 10,4 & 5,6 & 7,8 & 2,2 & 73 & 84 & 80 & -4 & & \\
12 & T12 & E & 5 & -2 & 3,9 & 5,9 & 4,4 & 0,6 & 2,5 & 1,9 & 81 & 100 & 92 & -8 & 0 & 2 \\
Média & & & 19,4 & 5,2 & 7,9 & 2,7 & 7,5 & $-3,7$ & 0,1 & 3,8 & 60,3 & 87,5 & 81,4 & $-6,1$ & 6,2 & 1,6 \\
\hline
\end{tabular}

ODI - Oswestry Disability Index; VAS - Visual analogic Scale.

Um paciente abandonou a consulta aos dois meses e dois não compareceram à avaliação clínica.

A angulação vertebral pré-operatória média foi de $19,4^{\circ}$. Obteve-se uma correcção de $14,2^{\circ}$ registando-se uma perda final de $2,7^{\circ}$.

A deformidade cifótica pré-operatória foi de $7,5^{\circ}$. Foi conseguida uma correcção média da cifose de $11,2^{\circ}$, melhorando a angulação cifótica para $3,7^{\circ}$. Na avaliação final, perdeu-se $3,8^{\circ}$ de correcção cifótica.

A percentagem de altura anterior do corpo vertebral pré-operatória foi de $60,3 \%$, tendo-se obtido uma correcção para $87,5 \%$. No seguimento, perdeu-se $6,1 \%$ de altura anterior em relação ao valor pós-operatório.

O resultado médio do Oswestry Disability Index (ODI) foi de 6,2 (de 0 a 18) e o da VAS de 1,6 (de 0 a 5).

À data da última consulta, nove pacientes foram classificados como nível E de Frankel e os dois pacientes com lesões neurológicas melhoraram de um nível B para D de Frankel.

Houve necessidade de realizar o reposicionamento de um parafuso pedicular. Registou-se um seroma pós-operatório com consequente drenagem cirúrgica e antibioterapia empírica. Não se registou nenhum caso de desmontagem ou falência de material. Não houve nenhuma lesão radicular ou dural.

\section{DISCUSSÃO}

Desde a sua introdução por Roy-Camille nos anos 1970 que a fixação vertebral com parafusos pediculares é utilizada no tratamento dessas fracturas ${ }^{20}$. Aliás, ainda hoje no seu serviço, se procura incluir na instrumentação o nível fracturado.

É já adquirido que a fixação pedicular com parafusos e barras confere uma excelente estabilidade multidireccional e por isso a sua aplicação encontra-se amplamente difundida. Contudo, o número de níveis a instrumentar no sentido de garantir a máxima estabilidade com o mínimo de compromisso da mobilidade permanece um dos pontos mais polémicos.

A fixação curta constitui uma técnica popular, uma vez que compromete minimamente a mobilidade e está associada a uma menor morbilidade potencial. Na literatura, porém, está relatada uma elevada taxa de falência da montagem e de perda de correcção ${ }^{12,21}$.

Estão descritos diferentes comprimentos de montagem. Katonis et al. ${ }^{22}$ verificaram que uma instrumentação dois níveis acima e um nível abaixo da fractura garantiria uma construção rígida e sem perda de correcção. Carl et al. ${ }^{15}$ afirmaram que a instrumentação pedicular dois níveis acima da fractura deveria ser usada na transição toracolombar, e Alvine et al. ${ }^{4}$ apontaram a vantagem de uma construção de dois níveis acima e dois abaixo em termos de controlo de estabilidade e redução da deformidade cifótica.

Recentemente, foram publicados estudos com montagens curtas, incluindo a instrumentação pedicular do nível fracturado ${ }^{23-25}$. Mahar et al. ${ }^{17}$ constataram, por meio de estudos biomecânicos em cadáveres, que a fixação segmentar curta incluindo o nível fracturado traduz-se numa acrescida estabilidade biomecânica, principalmente com a aplicação de stress em torsão axial. Propuseram igualmente que a instrumentação do nível fracturado constituiria um ponto adicional de fixação permitindo desse modo uma melhor redução e correcção de cifose. A aplicação clínica dessa premissa em 12 pacientes com um seguimento médio de 4,4 meses traduziu-se numa correcção cifótica de $15^{\circ}$, valor este superior aos obtidos com construções não-segmentares, tendo atingido valores finais de correcção cifótica de $10^{\circ}$, e uma perda de $5^{\circ}$ desde o pós-operatório imediato. Comparativamente, Carl et al. atingiram $7^{\circ}$ no pós-operatório ${ }^{15}$ enquanto Cho et al. obtiveram $6^{0}{ }^{26}$. Nesses dois últimos estudos referidos, grande parte da correcção cifótica foi perdida durante o tempo de seguimento.

McLain et al. demonstram que a maioria dos pacientes com instabilidade residual da coluna anterior geralmente 
desenvolvem deformidade progressiva nos primeiros seis meses pós-operatório ${ }^{12}$. Parece-nos, portanto, que o nosso seguimento de 14,5 meses será adequado para retirar conclusões deste estudo.

Obtivemos, no pós-operatório imediato, uma melhoria da angulação vertebral de $14,2^{\circ}$, da deformidade cifótica de $11,2^{\circ}$ e $27,2 \%$ de recuperação da altura vertebral anterior, verificando-se, ao tempo de seguimento final, perdas de $2,7^{\circ}, 3,8^{\circ}$ e $6,1 \%$, respectivamente.

Constatamos, no entanto, que a média da angulação vertebral inicial, $19,4^{\circ}$, é baixa. Tal facto deve a sua explicação à inclusão de dois pacientes nos quais se observou uma medição radiográfica quase normal da angulação vertebral inicial, mas que apresentavam défices neurológicos à admissão (Frankel B), e de um outro paciente com envolvimento do canal vertebral superior a $50 \%$, o que motivou a indicação cirúrgica.

Korovessis et al. ${ }^{27}$ constituíram dois grupos de estudo em que em um incluíam pacientes submetidos a cirurgia combinada, anterior e posterior, e no segundo grupo pacientes submetidos a fixação pedicular curta com instrumentação do nível fracturado. Neste último, registou-se uma perda de correcção cifótica de $5^{\circ}$, o que levou os autores a concluir que a montagem segmentar curta com instrumentação do nível fracturado não impedia a progressão da deformidade cifótica e, portanto, não seria opção no tratamento de fracturas do segmento médio lombar. Paradoxalmente, foram obtidos melhores resultados clínicos no grupo submetido a montagem segmentar curta com instrumentação do nível fracturado, apesar da progressão para cifose $\mathrm{e}^{28-29}$.

Mais tarde, Esses $^{30}$ realizou uma análise dos dados com critérios de Medicina Baseada em Evidência, tendo concluído que clinicamente não havia diferença entre os dois grupos, pelo que não se justificaria a morbilidade acrescida de uma via anterior. Os scores funcionais obtidos em nosso estudo permitiram-nos constatar o excelente resultado clínico de uma via posterior isolada. A via anterior, além de ser menos familiar para a maioria dos cirurgiões, constitui um procedimento indesejável nos politraumatizados com trauma abdominal associado ${ }^{17}$.

Do total de pacientes deste estudo, $17 \%$ apresentavam défices neurológicos à entrada, valor dentro do intervalo habitualmente referido na literatura ${ }^{31}$. Nesses casos, pode ser necessário realizar pediculectomia e hemilaminectomia para correcção do muro vertebral, procurando preservar ao máximo a coluna posterior, o que foi feito em um dos pacientes.

A instrumentação do nível fracturado aumenta a rigidez da montagem, protege a vértebra fracturada de cargas anteriores e garante um ponto de fixação adicional que permite melhor correcção por moldagem in situ ${ }^{17}$, a qual deve ser feita lentamente e apenas quando temos boa presa pedicular. Num osso osteoporótico é desaconselhado, mas não contraindicado.

\section{CONCLUSÃO}

Com base na literatura recente e de acordo com os resultados obtidos, a montagem curta com instrumentação do nível fracturado e moldagem in situ no tratamento das fracturas tipo burst da coluna toracolombar é uma técnica segura, realizada por uma abordagem familiar à maioria dos ortopedistas, a qual permite uma excelente redução que se mantém ao longo do tempo, registando-se apenas uma perda mínima sem significado clínico.

\section{REFERÊNCIAS}

1. Esses SI, Botsford DJ, Kostuik JP. Evaluation of surgical treatment for burst fractures. Spine (Phila Pa 1976). 1990;15(7):667-73.

2. Wood K, Buttermann G, Mehbod A, Garvey T, Jhanjee R, Sechriest V, et al. Operative compared with nonoperative treatment of a thoracolumbar burst fracture without neurological deficit. A prospective, randomized study. J Bone Joint Surg Am. 2003;85(5):773-81.

3. Denis F. The three column spine and its significance in the classification of acute thoracolumbar spinal injuries. Spine (Phila Pa 1976). 1983;8(8):817-31.

4. Alvine GF, Swain JM, Asher MA, Burton DC. Treatment of thoracolumbar burst fractures with variable screw placement or Isola instrumentation and arthrodesis: case series and literature review. J Spinal Disord Tech. 2004;17(4):251-64.
5. Briem D, Lehmann W, Ruecker AH, Windolf J, Rueger JM, Linhart W. Factors influencing the quality of life after burst fractures of the thoracolumbar transition. Arch Orthop Trauma Surg. 2004;124(7):461-8.

6. Dimar JR 2nd, Wilde PH, Glassman SD, Puno RM, Johnson JR.

Thoracolumbar burst fractures treated with combined anterior and posterior surgery. Am J Orthop (Belle Mead NJ). 1996;25(2):159-65.

7. Leferink VJ, Zimmerman KW, Veldhuis EF, ten Vergert EM, ten Duis HJ. Thoracolumbar spinal fractures: radiological results of transpedicular fixation combined with transpedicular cancellous bone graft and posterior fusion in 183 patients. Eur Spine J. 2001;10(6):517-23.
8. Parker JW, Lane JR, Karaikovic EE, Gaines RW. Successful shortsegment instrumentation and fusion for thoracolumbar spine fractures: a consecutive 41/2-year series. Spine (Phila Pa 1976). 2000;25(9):1157-70.

9. Holdsworth F. Fractures, dislocations, and fracture-dislocations of the spine. J Bone Joint Surg Am. 1970;52(8):1534-51.

10. Benson DR, Burkus JK, Montesano PX, Sutherland TB, McLain RF. Unstable thoracolumbar and lumbar burst fractures treated with the $\mathrm{AO}$ fixateur interne. J Spinal Disord Tech. 1992;5(3):335-43.

11. Esses SI, Botsford DJ, Wright T, Bednar D, Bailey S. Operative treatment of spinal fractures with the AO internal fixator. Spine (Phila $\mathrm{Pa}$ 1976). 1991;16(3 Suppl):146-50. 
12.McLain FR, Sparling E, Benson RD. Early failure of short-segment pedicle instrumentation for thoracolumbar burst fractures: a preliminary report. J Bone Joint Surg Am. 1993;75(2): 162-7.

13.Gurwitz GS, Dawson J, McNamara MJ, Federspiel CF, Spengler DM. Biomechanical analysis of three surgical approaches for lumbar burst fractures using short segment instrumentation. Spine (Phila Pa 1976). 1993;18(8):977-82.

14. Kramer DL, Rodgers WB, Mansfield FL. Transpedicular instrumentation and short-segment fusion of thoracolumbar fractures: a prospective study using a single instrumentation system. J Orthop Trauma. 1995;9(6):499-506.

15.Carl AL, Tromanhauser SG, Roger DJ. Pedicle screw instrumentation for thoracolumbar burst fractures and fracture-dislocations. Spine (Phila Pa 1976). 1992;17(8 Suppl):S317-24.

16.McNamara MJ, Stephens GC, Spengler DM. Transpedicular short-segment fusions for treatment of lumbar burst fractures. J Spinal Disord. 1992;5(2):183-7.

17. Mahar A, Kim C, Wedemeyer M, Mitsunaga L, Odell T, Johnson B, et al. Short-segment fixation of lumbar burst fractures using pedicle fixation at the level of the fracture. Spine (Phila Pa 1976). 2007;32(14):1503-7.

18.Denis F. Spinal instability as defined by the three-column spine concept in acute spinal trauma. Clin Orthop Relat Res. 1984;(189):65-76.

19.Frankel HL, Hancock DO, Hyslop G, Melzak J, Michaelis LS, Ungar GH, et al. The value of postural reduction in the initial management of closed injuries of the spine with paraplegia and tetraplegia. I. Paraplegia. 1969;7(3):179-92.
20.Roy-Camille R, Saillant G, Mazel C. Plating of thoracic, thoracolumbar, and lumbar injuries with pedicle screw plates. Orthop Clin North Am. 1986;17(1):147-59.

21.Ferguson RL, Tencer AF, Woodard P, Allen BL Jr. Biomechanical comparisons of spinal fracture models and the stabilizing effects of posterior instrumentations. Spine (Phila $\mathrm{Pa}$ 1976). 1988;139(5):453-60.

22.Katonis PG, Kontakis GM, Loupasis GA, Aligizakis AC, Christoforakis JI, Velivassakis EG. Treatment of unstable thoracolumbar and lumbar spine injuries using Cotrel-Dubousset instrumentation. Spine (Phila Pa 1976). 1999;24(22):2352-7.

23.Dick JC, Zdeblick TA, Bartel BD, Kunz DN. Mechanical evaluation of cross-link designs in rigid pedicle screw systems. Spine (Phila Pa 1976). 1997;22(4):370-5.

24.Leferink VJ, Nijboer JM, Zimmerman $\mathrm{KW}$, Veldhuis EF, tenVergert EM, ten $\mathrm{DH}$. Thoracolumbar spinal fractures: segmental range of motion after dorsal spondylodesis in 82 patients: a prospective study. Eur Spine J. 2002;11(1):2-7.

25.Kirkpatrick JS, Wilber RG, Likavec M, Emery SE, Ghanayem A. Anterior stabilization of thoracolumbar burst fractures using the Kaneda device: a preliminary report. Orthopedics. 1995;18(7):673-8.

26.Cho DY, Lee WY, Sheu PC.

Treatment of thoracolumbar burst fractures with polymethyl methacrylate vertebroplasty and short-segment pedicle screw fixation. Neurosurgery. 2003;53(6):1354-60; discussion 1360-1.
27.Korovessis P, Baikousis A, Zacharatos S, Petsinis G, Koureas G, Iliopoulos P. Combined anterior plus posterior stabilization versus posterior shortsegment instrumentation and fusion for mid-lumbar (L2-L4) burst fractures. Spine (Phila Pa 1976). 2006;31(8):859-68.

28.Korovessis PG. Re: Mahar A, Kim C, Wedemeyer M, et al. Shortsegment fixation of lumbar burst fractures using pedicle fixation at the level of the fracture. Spine 2007;32(14):1503-7. Spine (Phila Pa 1976). 2007;32(23):2638-9; author reply 2639.

29. Verlaan JJ, Oner FC. Re: Korovessis $\mathrm{P}$, Baikousis A, Zacharatos S, et al. Combined anterior plus posterior stabilization versus posterior short-segment instrumentation and fusion for mid-lumbar (L2-L4) burst fractures. Spine 2006;31:85968. Spine (Phila Pa 1976). 2006; 31(21):2521; author reply 2521-2.

30.Esses S. Posterior short-segment instrumentation and fusion provides better results than combined anterior plus posterior stabilization for midlumbar (12 to 14) burst fractures. J. Bone Joint Surg Am. 2006; 88(10):2311.

31.Benson DR. Unstable thoracolumbar fractures, with emphasis on the burst fracture. Clin Orthop Relat Res. 1988;(230):14-29.

\section{Correspondência}

Pedro Cacho Rodrigues

Alameda Prof. Hernâni Monteiro, 4200

Porto - Portugal

Tel: (+351) 225512100

E-mail: pedrocachorodrigues@gmail.com 